\title{
O acesso à informação sobre higiene bucal e as perdas dentárias por cárie entre adultos
}

\author{
Access to information on oral hygiene and tooth loss \\ due to caries among adults
}

Desirée Sant'Ana Haikal ${ }^{1}$

Andréa Maria Eleutério de Barros Lima Martins ${ }^{1}$

Pedro Henrique Soares Aguiar ${ }^{2}$

Marise Fagundes Silveira ${ }^{1}$

Alfredo Maurício Batista de Paula ${ }^{1}$

Efigênia Ferreira e Ferreira ${ }^{3}$

\footnotetext{
${ }^{1}$ Programa de PósGraduação em Ciências da Saúde, Universidade Estadual de Montes Claros. Av. Cula Mangabeira 562, Santo Expedito. 39.401-001 Montes Claros MG Brasil. desireehaikal@gmail.com ${ }^{2}$ Curso de Odontologia, Universidade Estadual de Montes Claros. ${ }^{3}$ Departamento de Odontologia Social e Preventiva, Faculdade de Odontologia, Universidade
}

Abstract This study sought to establish the association between tooth loss due to dental caries and variables related to access to information on oral health in adults aged $35-44$ years $(n=780)$ who participated in a large epidemiological study. The dependent variable was total tooth loss due to caries. The independent variables were grouped into blocks: demographic and socio-economic characteristics, use of dental service, access to oral health information (about how to prevent oral problems; oral hygiene; and diet), and behaviors. The analysis was conducted using hierarchical multiple linear regression. The mean number of lost teeth due to caries was 7.03. High numbers of lost teeth were higher among females and older adults; low levels of education; adults who rarely/ never received oral hygiene information; those who brushed their teeth once a day or less; and those adults who did not use dental floss $(p<$ $0.05)$. Adults who rarely/never received oral hygiene information from dental services lost 2.15 more teeth due to caries than those who always/ often received such information $(p=0,000)$. Thus, it is suggested that access to information should be encouraged to contribute to greater equity in oral health.

Key words Adults, Tooth loss, Health education, Oral health, Epidemiology
Resumo Objetivou-se testar a associação entre perdas dentárias por cárie (PDC) e variáveis relativas ao acesso a informações em saúde bucal. Foram analisados dados de 780 adultos (35-44 anos) participantes de um estudo epidemiológico. A variável dependente foi o total de PDC e as independentes foram reunidas em blocos de variáveis: demográficas e socioeconômicas; utilização dos serviços odontológicos; acesso a informações em saúde bucal (informações sobre como evitar problemas bucais, sobre higiene bucal e sobre dieta) e; comportamentais. Conduziu-se regressão linear múltipla hierarquizada. O número médio de PDC foi de 7,03 (EP=0,31) e 83\% dos adultos haviam perdido algum dente por cárie. As PDC foram maiores entre os mais velhos, os com menor escolaridade, as mulheres, os que raramente ou nunca receberam informações sobre higiene bucal, os que escovavam os dentes uma vez ou menos ao dia e entre os que não usavam fio dental. Adultos que raramente/nunca receberam informações dos serviços odontológicos sobre higiene bucal perderam 2,15 dentes a mais por cárie que aqueles que sempre/frequentemente receberam tais informações $(p=0,000)$. Assim, sugerese que a garantia do acesso à informação deva ser incentivada a fim de contribuir com maior equidade em saúde bucal.

Palavras-chave Adultos, Perda de dente, Educação em saúde, Saúde bucal, Epidemiologia 
Introdução

Educação em saúde refere-se a quaisquer combinações de experiências de aprendizagem delineadas com vistas a facilitar ações voluntárias relativas à saúde ${ }^{1}$. Dentre as estratégias de educação em saúde, a oferta de informação é apontada como elemento chave ${ }^{2}$. Desfechos favoráveis almejados pela educação em saúde incluem desde maior conhecimento e entendimento dos pacientes sobre suas condições; sobre suas percepções e experiências da doença e tratamento; até impactos sobre o comportamento de saúde e, consequentemente, no estado de saúde ${ }^{2}$. Revisões sistemáticas têm revelado evidências da eficácia de tais abordagens, considerando diversos temas relacionados à saúde, entre eles obesidade ${ }^{3}$, câncer ${ }^{4}$, atividade física ${ }^{5}$ e doenças crônicas ${ }^{6}$. Outras revisões, no entanto, encontraram pequena evidência da efetividade de programas informativos sobre a mudança individual de comportamento ${ }^{7,8}$.

$\mathrm{Na}$ odontologia, a educação em saúde tem sido parte importante da assistência, sendo os serviços de saúde cenários constantes dessa prática. Já foi observado impacto de medidas educativas na redução de placa e gengivite, na melhora da condição de higiene, na halitose, no conhecimento e até mesmo na satisfação do paciente $^{9-11}$. Entretanto, os efeitos da educação em saúde sobre a cárie e a perda de dentes não foi suficientemente explorado previamente.

Um dos principais indicadores de saúde bucal em população adulta é a perda dentária, haja vista que é considerada como fracasso de medidas preventivas e/ou curativas prévias ${ }^{12-14}$. Por ser evitável na maioria dos casos e acarretar importantes prejuízos na vida das pessoas ${ }^{12,15,16}$ a perda dentária representa um desafio para a saúde pública ${ }^{17}$. Muitos estudos apontam programas preventivos de educação em saúde para minimizar a perda dentária ${ }^{14,18-20}$, embora não tenha sido localizado um estudo que tenha investigado se tais abordagens estariam, de fato, associadas a menor número de perdas dentárias.

Considerando que é na fase adulta da vida que maior número de dentes são perdidos ${ }^{21}$, este estudo objetivou estimar a prevalência de perdas dentárias por cárie entre adultos e testar a associação entre tais perdas e variáveis relativas ao acesso à informações em saúde bucal.

\section{Metodologia}

Foram utilizados dados relativos aos adultos participantes do levantamento epidemiológico em saúde bucal "Projeto SBMOC" realizado no município de Montes Claros, situado no norte do estado de Minas Gerais, Brasil, em 2008/2009. Detalhes da metodologia adotada encontram-se em estudo prévio ${ }^{22}$. Destacam-se aqui, os principais aspectos metodológicos adotados:

Amostragem: Complexa, probabilística, por conglomerados em dois estágios (setores censitários e quadras), garantindo proporcionalidade por sexo. Para a população de 35-44 anos, grupo padrão para avaliação das condições de saúde bucal em adultos ${ }^{23}$, os cálculos evidenciaram a necessidade de se avaliar 762 indivíduos, considerando uma prevalência estimada em 0,50 do evento estudado (cárie dentária), nível de confiança (95\%) e erro amostral (5,5\%). Para a correção do efeito de desenho, adotou-se deffigual a 2,0. Foi também estabelecido um acréscimo de $20 \%$ para compensar possíveis perdas.

Calibração dos examinadores: Participaram da coleta de dados do Projeto SBMOC, 24 cirurgiões-dentistas treinados e calibrados, que alcançaram concordância Kappa satisfatória ${ }^{24}$. Os mesmos foram acompanhados por anotadores/ digitadores treinados. Foram adotados os critérios de diagnóstico da $4^{a}$ edição do Oral Health Surveys: Basic Methods, da Organização Mundial da Saúde (OMS) ${ }^{23}$.

Coleta de dados: No domicílio, após assinatura do termo de consentimento livre e esclarecido, foram realizadas entrevistas e exames intrabucais. Os exames foram conduzidos sob luz natural, com auxílio de espelho e adotando todos os códigos/critérios propostos pela $\mathrm{OMS}^{23}$. Para composição do índice CPOD (número total de dentes cariados, perdidos e restaurados), as condições das coroas dentárias foram avaliadas e os elementos perdidos/extraídos receberam os códigos 4 (perda dentária por cárie) ou 5 (perda dentária por outra razão que não a cárie ${ }^{23}$. Os dados foram registrados em computadores de mão, utilizando um programa criado especificamente para esse fim.

\section{Análise estatística}

As análises foram realizadas empregando-se o programa estatístico PASW (Predictive Analytics Software - SPSS ${ }^{\oplus}$ ) e foram conduzidas respeitando-se a necessidade de correção pelo efeito de desenho por serem proveniente de amostras 
por conglomerados. Os 11 indivíduos (1,3\%) que relataram nunca terem ido ao dentista foram excluídos das análises.

A variável dependente foi o número total de perdas dentárias por cárie, construída a partir da soma dos códigos 4 do índice CPOD para o diagnóstico de cada um dos 32 espaços dentários examinados por indivíduo. A mesma foi trabalhada conforme sua natureza numérica discreta. As variáveis independentes foram reunidas em quatro blocos de variáveis, sendo:

Bloco 1- Demográficas e socioeconômicas: Idade, anos de estudo, renda per capita, sexo (masculino; feminino) e cor da pele autodeclarada (negros/pardos; brancos). Idade, anos de estudo e renda foram mantidas como variáveis numéricas.

Bloco 2 - Utilização dos serviços odontológicos: Tipo de serviço utilizado (privado; público), tempo desde a última consulta em anos $(<1 ; 1-2$; $>3$ ) e uso regular/periódico (sim; não).

Bloco 3 - Acesso a informações em saúde bucal: informações sobre como evitar problemas bucais (sim; não), informações sobre higiene bucal (sempre/frequentemente; ocasionalmente; raramente/nunca) e informações sobre dieta (sempre/frequentemente; ocasionalmente; raramente/nunca). Tais variáveis foram obtidas, respectivamente, das seguintes perguntas: Recebeu informações sobre como evitar problemas bucais?; Recebeu orientações sobre higiene bucal nos serviços odontológicos? e Recebeu orientações sobre dieta nos serviços odontológicos?

Bloco 4 - Comportamentais: hábito tabagista (ausente; presente), número de escovações diárias $(>3 ; 2 ;<1)$ e uso de fio dental (usa; não usa).

Inicialmente foi realizada análise exploratória da variável dependente, estimando sua média e sua proporção em função do total de perdas dentárias (código $4+$ código 5). Foi estimada a proporção de indivíduos segundo o total de perdas dentárias por cárie. A seguir, em cada bloco, conduziu-se análise descritiva de todas as variáveis. Para as variáveis independentes categóricas foram apresentados números absolutos e percentuais corrigidos pelo efeito de desenho, bem como foi estimada média e erro-padrão do total de perdas dentárias por cárie para cada categoria. Para as variáveis independentes numéricas foram estimadas médias e seus erros-padrão também corrigidos.

Foi realizada análise univariada da variável dependente com cada uma das variáveis independentes através da regressão linear simples (modelo simples). As variáveis categóricas com três categorias foram transformadas em variáveis Dummy. A magnitude das associações foi apresentada pelos parâmetros estimados do coeficiente da regressão linear múltipla $(\beta)$, seus respectivos erros-padrão e valor p. Todas as variáveis independentes que se mostraram associadas à dependente ao nível de $20 \%(\mathrm{p}<0,2)$ foram selecionadas para comporem modelos múltiplos em cada bloco de variáveis.

Para construção do modelo múltiplo em cada bloco, adotou-se o procedimento passo a passo (stepwise backward), de forma que os modelos foram sendo ajustados, removendo-se, uma a uma, as variáveis que não se mostraram associadas à variável dependente $(\mathrm{p}<0,05)$. O percentual da variância explicada pelo modelo foi calculado através do coeficiente de determinação ajustado ( $\mathrm{R}^{2}$ ajustado).

Os blocos de variáveis compuseram o modelo hierarquizado adotado para perdas dentárias por cárie (Figura 1). A composição dos blocos, bem como a ordem de entrada dos mesmos na modelagem hierarquizada baseou-se em modelos prévios, englobando reconhecidos fatores de risco para problemas bucais ${ }^{12,17,25,26}$. As variáveis mantidas no modelo múltiplo de cada bloco compuseram a análise de regressão linear múltipla hierarquizada. O bloco de variáveis Demográficas e socioeconômicas (Bloco 1) foi o primeiro a ser incluído no modelo, permanecendo como fator de ajuste para os demais blocos. Em seguida foram incluídas as variáveis de Utilização dos serviços odontológicos (Bloco 2), permanecendo no modelo somente aquelas que apresentaram nível descritivo $\mathrm{p}<0,05$ após o ajuste, repetindose o processo, da mesma maneira, com as variáveis Acesso a informações em saúde bucal (Bloco 3) e Comportamentais (Bloco 4). A magnitude das associações entre a variável dependente e os fatores de interesse, em cada bloco, foi apresentada pelos parâmetros estimados do coeficiente da regressão linear múltipla $(\beta)$, seus respectivos erros-padrão e valor $\mathrm{p}$.

Os pressupostos para a regressão linear foram testados: linearidade dos parâmetros, homocedasticidade (variância constante de erros), independência dos termos (covariância nula), normalidade na distribuição dos resíduos e ausência de multicolinearidade. A linearidade foi avaliada pela análise gráfica de resíduos do diagrama de dispersão e pelo coeficiente de correlação. O diagnóstico da homocedasticidade foi feito a partir da análise gráfica de resíduos. A independência dos termos foi verificada pelo teste Durbin-Watson (DW). A normalidade da distri- 


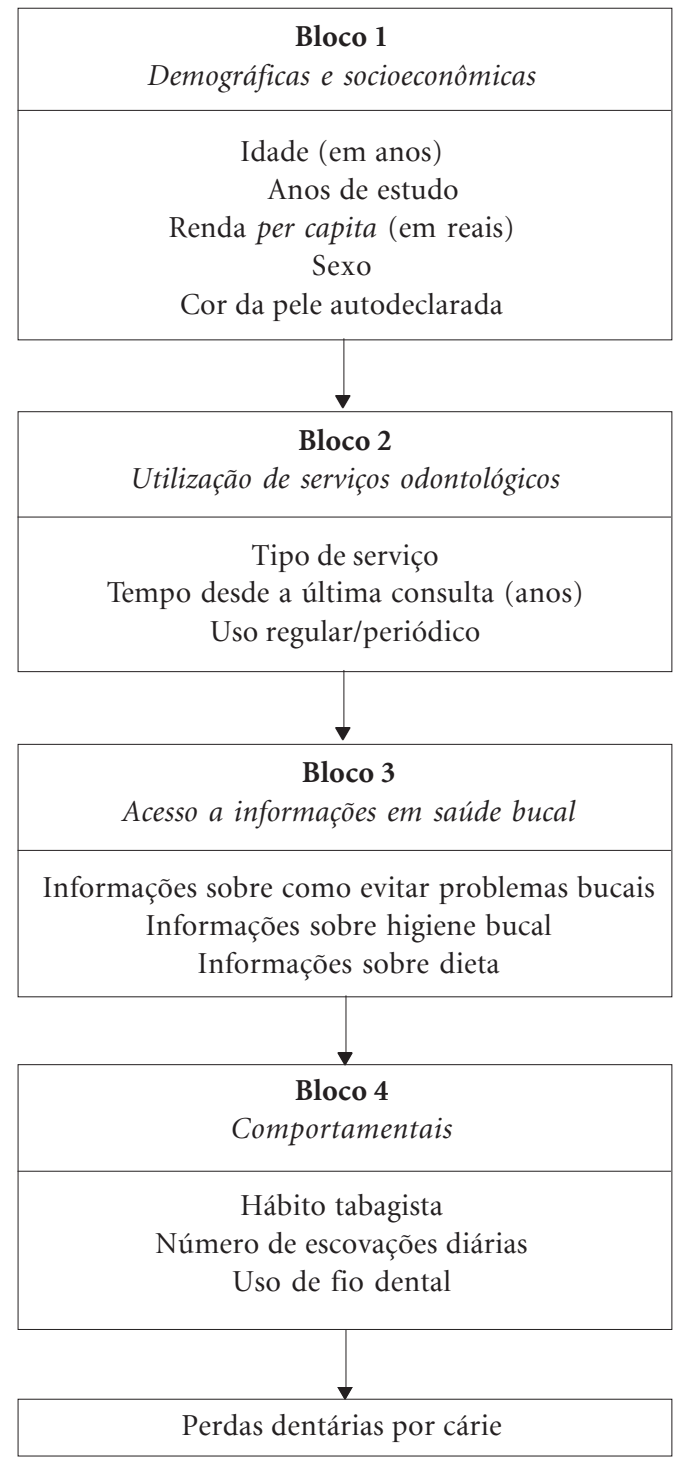

Figura 1. Modelo hierarquizado adotado para perdas dentárias por cárie.

buição dos resíduos foi testada por meio do gráfico de probabilidade normal para os resíduos e por meio dos testes de aderência à normalidade Kolmogorov-Smirnov (KS) com correção de Shapiro-Wilks. A multicolinearidade foi avaliada pela análise do valor da tolerância e seu inverso, o fator de inflação de variância $(\mathrm{VIF}=1$ / tolerância). Um valor de referência comumente usado é tolerância de 0,10 , o que corresponde a um VIF acima de $10^{27,28}$.

O "Projeto SBMOC" recebeu aprovação do Comitê de Ética em Pesquisas da Unimontes. Os participantes foram devidamente informados sobre a pesquisa e concordaram participar da mesma através da assinatura do termo de consentimento livre e esclarecido.

\section{Resultados}

Entre os 780 adultos incluídos neste estudo, observou-se que as perdas por cárie representaram $86 \%$ do total de perdas dentárias. Verificou-se uma média de $8,2(\mathrm{EP}=0,37)$ dentes perdidos por adulto, sendo que a média de perdidos por cárie foi de 7,03 (EP = 0,31, Deff=1,38). A proporção de indivíduos adultos segundo o número total de perdas dentárias por cárie foi heterogênea, sendo que $83,2 \%$ dos adultos já haviam sofrido pelo menos uma perda por cárie (Figura 2).

$\mathrm{Na}$ análise descritiva percebeu-se renda per capita média baixa, sendo que 611 indivíduos (78\%) viviam com menos de um salário mínimo vigente, que em 2009 era de R $\$ 465,00$ ou US\$ 245.00. O tempo médio de estudo foi inferior a 10 anos, sendo que cerca de $15 \%$ possuía até 4 anos e $25 \%$ possuía 12 ou mais anos de estudo. Quanto ao acesso às informações em saúde bucal, quase dois terços alegaram ter recebido informações sobre como evitar problemas bucais, menos da metade alegou ter recebido informações frequentes sobre higiene bucal e menos de um quinto quanto à dieta (Tabela 1 ). O número médio de perdas dentárias por cárie em cada categoria investigada também pode ser visualizado na Tabela 1.

Nas análises univariadas, todas as variáveis independentes, exceto hábito tabagista, apresentaram associação com o número de perdas dentárias por cárie ao nível de 20\% (modelo simples, Tabela 2). Todas as demais variáveis foram selecionadas para iniciar a composição dos modelos múltiplos. A Tabela 2 apresenta os modelos múltiplos ajustados em cada bloco de variáveis. No bloco 1 a variável cor da pele autodeclarada perdeu associação com a variável dependente. Os blocos 2 e 4 permaneceram com todas as variáveis associadas à dependente. No bloco 3, as variáveis informações sobre como evitar problemas bucais e sobre dieta perderam associação com a variável dependente. Somente a variável informações sobre higiene bucal manteve-se no bloco 3 e esta, por si só, foi capaz de explicar $2,3 \%$ do total de perdas dentárias por cárie.

A Tabela 3 apresenta a análise de regressão linear múltipla hierarquizada. No modelo final (modelo 4), o total de perdas dentárias por cárie foi maior entre os adultos mais velhos, com me- 


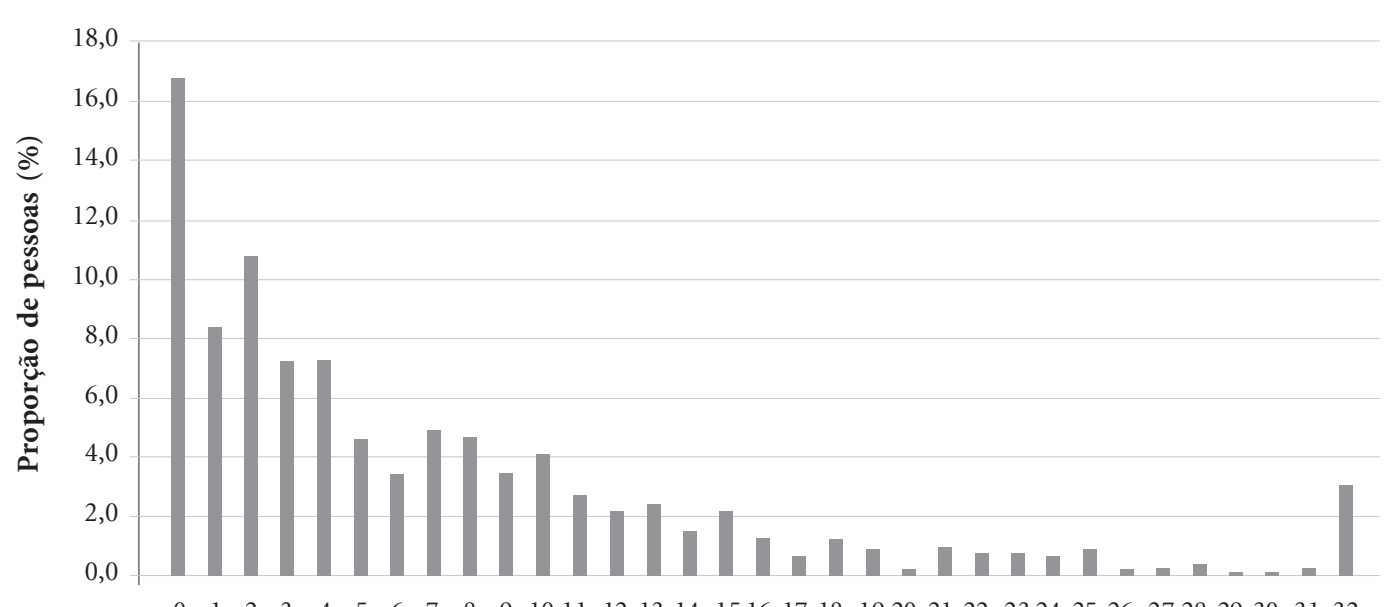

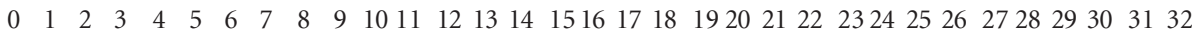

Total de perdas dentárias por cárie

Figura 2. Proporção de indivíduos adultos segundo o total de perdas dentárias por cárie. Projeto SBMOC, 2009.

nos anos de estudo, do sexo feminino, que raramente ou nunca receberam informações sobre higiene bucal, que escovavam os dentes uma vez ou menos ao dia e entre os que não usavam fio dental ( $\mathrm{p}>0,05)$. Este modelo foi capaz de explicar $27,5 \%$ da variabilidade do total de perdas dentárias por cárie. As variáveis Demográficas e socioeconômicas foram as que mais contribuíram na explicação da variável dependente $\left(\mathrm{R}^{2}\right.$ ajustado $=23,5 \%$, modelo 1 ). Nenhuma variável de Utilização dos serviços odontológicos mantevese associada à dependente após ajuste pelos outros blocos. A frequência de acesso a informações sobre higiene bucal manteve-se associada ao total de perdas dentárias por cárie, mesmo após todos os ajustes pelos outros blocos de variáveis. Adultos que raramente/nunca receberam informações dos serviços odontológicos sobre higiene bucal perderam 2,15 dentes a mais por cárie que aqueles que sempre/frequentemente receberam tais informações $(\mathrm{p}=0,000)$. Já aqueles que receberam essas informações ocasionalmente, perderam 1,41 dentes a mais também quando comparados aos que as receberam sempre/frequentemente $(\mathrm{p}=0,056)$.

Nos testes dos pressupostos para a regressão linear, foram verificados linearidade dos parâmetros $(r<0,3)$, independência dos termos (DW $=2,062$, p d" 0,05$)$ e ausência de multicolinearidade (tolerâncias $>0,10$ e VIF < 10). Houve vio- lação dos pressupostos de homocedasticidade e de normalidade na distribuição dos resíduos $(\mathrm{KS}=2,462, \mathrm{p}=0,000)$.

\section{Discussão}

Este estudo mostrou elevada prevalência de perdas dentárias entre os adultos, embora enquadrada numa tendência de queda. A média de 8,2 dentes perdidos encontrada foi abaixo da verificada em 2002/2003 $(13,2)^{29}$ e um pouco acima da verifica em $2010(7,48)^{30}$ entre adultos brasileiros, mas ainda considerada alta perto de países desenvolvidos como a Irlanda $(5,7$ no ano $2000)^{31}$. A tendência de queda deverá se acentuar nos próximos anos diante do efeito de coorte do acesso ao flúor, que atingiu a população brasileira de forma expressiva somente a partir dos anos $90^{17}$ quando os adultos investigado possuíam aproximadamente 16-25 anos de idade e possivelmente já haviam sido acometidos pela cárie dentária. Espera-se, então, que a partir de 2018 os efeitos do flúor sejam percebidos de forma mais nítida entre adultos de 35-44 anos.

Os critérios de diagnóstico propostos pela OMS para levantamentos epidemiológicos consideram somente duas opções para a condição de coroas perdidas: perdidas por cárie (código 4) ou perdidas por outras razões (código 5$)^{23}$ 
Tabela 1. Distribuição dos adultos em números absolutos (n), percentuais (\%), média ( $\mu$ ) e erro padrão (EP) corrigidos pelo efeito de desenho, relativos ao número de perdas dentárias por cárie. Projeto SBMOC, 2009.

\begin{tabular}{|c|c|c|c|}
\hline \multirow[b]{2}{*}{ Variáveis } & \multicolumn{2}{|c|}{ Adultos } & \multirow{2}{*}{$\begin{array}{c}\begin{array}{c}\text { Total de perdas } \\
\text { dentárias por cárie }\end{array} \\
\mu(\mathrm{EP})\end{array}$} \\
\hline & $\mathbf{n}$ & $\%$ & \\
\hline \multicolumn{4}{|l|}{ Demográficas e socioeconômicas } \\
\hline Idade $(\text { em anos })^{\S}$ & \multicolumn{3}{|c|}{$\mu(\mathrm{EP})=39,50(0,13)$} \\
\hline Anos de estudo & \multicolumn{3}{|c|}{$\mu(\mathrm{EP})=9,17(0,32)$} \\
\hline Renda per capita (em reais) ${ }^{\S}$ & \multicolumn{3}{|c|}{$\mu(E P)=317,12(22,91)$} \\
\hline \multicolumn{4}{|l|}{ Sexo } \\
\hline Masculino & 362 & 47,9 & $6,23(0,43)$ \\
\hline Feminino & 418 & 52,1 & $7,80(0,51)$ \\
\hline \multicolumn{4}{|l|}{ Cor da pele autodeclarada } \\
\hline Brancos & 228 & 28,1 & $6,01(0,50)$ \\
\hline Negros/Pardos & 552 & 71,9 & $7,43(0,35)$ \\
\hline \multicolumn{4}{|c|}{ Utilização dos serviços odontológicos } \\
\hline \multicolumn{4}{|l|}{ Tipo de Serviço } \\
\hline Privado & 508 & 65,4 & $6,32(0,40)$ \\
\hline Público & 272 & 34,6 & $8,37(0,54)$ \\
\hline \multicolumn{4}{|c|}{ Tempo desde a última consulta (anos) } \\
\hline$<1$ & 350 & 45,5 & $6,00(0,41)$ \\
\hline $1-2$ & 209 & 24,2 & $6,10(0,43)$ \\
\hline$>3$ & 221 & 30,3 & $9,31(0,57)$ \\
\hline \multicolumn{4}{|l|}{ Uso regular/periódico ${ }^{\#}$} \\
\hline Sim & 151 & 18,7 & $4,51(0,45)$ \\
\hline Não & 628 & 81,3 & $7,58(0,35)$ \\
\hline \multicolumn{4}{|c|}{ Acesso a informações em saúde bucal } \\
\hline \multicolumn{4}{|c|}{ Informações sobre como evitar problemas bucais ${ }^{\#}$} \\
\hline $\operatorname{Sim}$ & 490 & 61,4 & $6,24(0,35)$ \\
\hline Não & 281 & 38,6 & $8,29(0,56)$ \\
\hline \multicolumn{4}{|c|}{ Informações sobre higiene bucal ${ }^{\#}$} \\
\hline Sempre/Frequentemente & 332 & 42,4 & $5,56(0,39)$ \\
\hline Ocasionalmente & 209 & 26,2 & $7,17(0,50)$ \\
\hline Raramente/Nunca & 237 & 31,4 & $8,86(0,66)$ \\
\hline \multicolumn{4}{|l|}{ Informações sobre dieta ${ }^{\#}$} \\
\hline Sempre/Frequentemente & 135 & 17,1 & $6,08(0,85)$ \\
\hline Ocasionalmente & 161 & 19,8 & $6,01(0,68)$ \\
\hline Raramente/Nunca & 480 & 63,1 & $7,56(0,44)$ \\
\hline \multicolumn{4}{|l|}{ Comportamentais } \\
\hline \multicolumn{4}{|l|}{ Habito tabagista } \\
\hline Ausente & 593 & 75,1 & $6,86(0,35)$ \\
\hline Presente & 187 & 24,9 & $7,53(0,76)$ \\
\hline \multicolumn{4}{|l|}{$\mathrm{N}^{\circ}$ escovações diárias ${ }^{\#}$} \\
\hline$>3$ & 491 & 64,3 & $5,92(0,35)$ \\
\hline 2 & 243 & 29,9 & $8,44(0,60)$ \\
\hline$<1$ & 44 & 5,8 & $11,38(1,40)$ \\
\hline \multicolumn{4}{|l|}{ Uso de fio dental } \\
\hline Usa & 405 & 50,3 & $4,90(0,36)$ \\
\hline Não usa & 375 & 49,7 & $9,65(055)$ \\
\hline
\end{tabular}

${ }^{\S}$ Variáveis numéricas. "Variação no $\mathrm{n}=780$ por perda de informações.

tais como doenças periodontais, traumas, dentes inclusos, indicações ortodônticas e protéticas, que são também causas de perdas dentárias $^{14,18-20}$. Nessa perspectiva, $86 \%$ das coroas per- didas foram registradas como perdidas por cárie, corroborando com estudos prévios que também encontraram altas prevalências de perdas por cárie considerando o total de perdas dentá- 
Tabela 2. Modelos de regressão linear simples e múltiplos para cada bloco de variáveis, relativos ao total de perdas dentárias por cárie entre adultos. Projeto SBMOC, 2009.

\begin{tabular}{|c|c|c|c|c|}
\hline & \multicolumn{2}{|c|}{ Modelo simples } & \multicolumn{2}{|c|}{ Modelo múltiplo } \\
\hline & $\beta(\mathbf{E P})$ & Valor $\mathrm{p}$ & $\beta(\mathrm{EP})$ & Valor $p$ \\
\hline \multicolumn{5}{|c|}{ Bloco 1 - Demográficas e socioeconômicas } \\
\hline Idade (em anos) & $0,83(0,12)$ & 0,000 & $0,68(0,08)$ & 0,000 \\
\hline Anos de estudo & $-0,74(0,07)$ & 0,000 & $-0,60(0,07)$ & 0,000 \\
\hline Renda per capita (em reais) & $-0,005(0,001)$ & 0,000 & $-0,002(0,001)$ & 0,040 \\
\hline \multicolumn{5}{|l|}{ Sexo } \\
\hline Masculino & ref. & & ref. & \\
\hline Feminino & $1,54(0,72)$ & 0,039 & $1,09(0,51)$ & 0,044 \\
\hline \multicolumn{5}{|l|}{ Cor da pele autodeclarada } \\
\hline Brancos & ref. & & & \\
\hline Negros/Pardos & $1,42(0,54)$ & 0,012 & n.s. & n.s. \\
\hline $\mathrm{R}^{2}$ ajustado & & & & $23,5 \%$ \\
\hline \multicolumn{5}{|c|}{ Bloco 2 - Utilização dos serviços odontológicos } \\
\hline \multicolumn{5}{|l|}{ Tipo de Serviço } \\
\hline Privado & ref. & 0,006 & ref. & \\
\hline Público & $2,05(0,70)$ & & $2,01(0,58)$ & 0,001 \\
\hline \multicolumn{5}{|c|}{ Tempo desde a última consulta (anos) } \\
\hline$<1$ & ref. & & & \\
\hline $1-2$ & $-0,09(0,53)$ & 0,858 & ref. & 0,489 \\
\hline$>3$ & $3,39(0,62)$ & 0,000 & $-0,47(0,68)$ & 0,001 \\
\hline \multicolumn{5}{|l|}{ Uso regular/periódico } \\
\hline Sim & ref. & & & \\
\hline Não & $3,74(0,59)$ & 0,000 & $2,27(0,70)$ & 0,006 \\
\hline $\mathrm{R}^{2}$ ajustado & & & & $6,4 \%$ \\
\hline \multicolumn{5}{|c|}{ Bloco 3 - Acesso a informações em saúde bucal } \\
\hline \multicolumn{5}{|c|}{ Informações sobre como evitar problemas bucais } \\
\hline Sim & ref. & & ref. & \\
\hline Não & $2,05(0,64)$ & 0,003 & $2,08(0,75)$ & n.s. \\
\hline \multicolumn{5}{|c|}{ Informações sobre higiene bucal } \\
\hline Sempre/Frequentemente & ref. & & ref. & \\
\hline Ocasionalmente & $1,60(0,68)$ & 0,024 & $1,60(0,68)$ & 0,024 \\
\hline Raramente/Nunca & $3,29(0,78)$ & 0,000 & $3,29(0,78)$ & 0,000 \\
\hline \multicolumn{5}{|l|}{ Informações sobre dieta } \\
\hline Sempre/Frequentemente & ref. & & & \\
\hline Ocasionalmente & $-0,68(1,20)$ & 0,950 & n.s & n.s \\
\hline Raramente/Nunca & $1,478(0,95)$ & 0,130 & n.s & n.s \\
\hline $\mathrm{R}^{2}$ ajustado & & & & $2,3 \%$ \\
\hline \multicolumn{5}{|l|}{ Bloco 4 - Comportamentais } \\
\hline \multicolumn{5}{|l|}{ Habito tabagista } \\
\hline Ausente & n.s & n.s & - & - \\
\hline Presente & n.s & n.s & - & - \\
\hline \multicolumn{5}{|l|}{$\mathrm{N}^{\circ}$ escovações diárias } \\
\hline$>3$ & ref. & & ref. & \\
\hline 2 & $2,51(0,70)$ & 0,001 & $1,86(0,60)$ & 0,002 \\
\hline$<1$ & $5,46(1,43)$ & 0,001 & $3,63(1,19)$ & 0,002 \\
\hline \multicolumn{5}{|l|}{ Uso de fio dental } \\
\hline Usa & ref. & & ref. & \\
\hline Não usa & $4,28(0,66)$ & 0,000 & $3,87(0,55)$ & 0,000 \\
\hline $\mathrm{R}^{2}$ ajustado & & & & $10,1 \%$ \\
\hline
\end{tabular}

ref. : categoria de referência. n.s. : não significativo. 
Tabela 3. Análise de regressão linear múltipla hierarquizada relativa ao total de perdas dentárias por cárie entre adultos. Projeto SBMOC, 2009.

\begin{tabular}{|c|c|c|c|c|}
\hline & \multicolumn{2}{|c|}{$\begin{array}{l}\text { Modelo } 1 \\
\text { (Bloco 1) }\end{array}$} & \multicolumn{2}{|c|}{$\begin{array}{c}\text { Modelo } 2 \\
(\text { Blocos } 1 \text { e } 2)\end{array}$} \\
\hline & $\beta(\mathrm{EP})$ & Valor $\mathrm{p}$ & $\beta(\mathrm{EP})$ & Valor $p$ \\
\hline \multicolumn{5}{|c|}{ Bloco 1 - Demográficas e socioeconômicas } \\
\hline Idade (em anos) & $0,68(0,08)$ & 0,000 & $0,66(0,08)$ & 0,000 \\
\hline Anos de estudo & $-0,60(0,07)$ & 0,000 & $-0,58(0,06)$ & 0,000 \\
\hline Renda per capita (em reais) & $-0,002(0,001)$ & 0,040 & n.s. & n.s. \\
\hline \multicolumn{5}{|l|}{ Sexo } \\
\hline Masculino & ref. & & ref. & \\
\hline Feminino & $1,09(0,51)$ & 0,044 & $1,30(0,49)$ & 0,009 \\
\hline \multicolumn{5}{|c|}{ Bloco 2 - Utilização dos serviços odontológicos } \\
\hline \multicolumn{5}{|l|}{ Tipo de Serviço } \\
\hline Privado & & & ref. & \\
\hline Público & & & $1,09(0,54)$ & 0,044 \\
\hline \multicolumn{5}{|c|}{ Tempo desde a última consulta (anos) } \\
\hline \multicolumn{5}{|l|}{$<1$} \\
\hline $1-2$ & & & n.s. & n.s. \\
\hline$>3$ & & & n.s. & n.s. \\
\hline \multicolumn{5}{|l|}{ Uso regular/periódico } \\
\hline \multicolumn{5}{|l|}{ Sim } \\
\hline Não & & & $1,41(0,66)$ & 0,032 \\
\hline \multicolumn{5}{|c|}{ Bloco 3 - Acesso a informações em saúde bucal } \\
\hline \multicolumn{5}{|c|}{ Informações sobre como higiene bucal ${ }^{\#}$} \\
\hline \multicolumn{5}{|l|}{ Sempre/Frequentemente } \\
\hline \multicolumn{5}{|l|}{ Ocasionalmente } \\
\hline \multicolumn{5}{|l|}{ Raramente/Nunca } \\
\hline \multicolumn{5}{|l|}{ Bloco 4 - Comportamentais } \\
\hline \multicolumn{5}{|l|}{$\mathrm{N}^{\circ}$ escovações diárias" } \\
\hline \multicolumn{5}{|l|}{$>3$} \\
\hline \multicolumn{5}{|l|}{2} \\
\hline \multicolumn{5}{|l|}{$<1$} \\
\hline \multicolumn{5}{|l|}{ Uso de fio dental } \\
\hline \multicolumn{5}{|l|}{ Usa } \\
\hline \multicolumn{5}{|l|}{ Não usa } \\
\hline $\mathrm{R}^{2}$ ajustado & $23,5 \%$ & & $23,6 \%$ & \\
\hline
\end{tabular}

rias $^{14,18,20,32}$. Entretanto, na prática de levantamentos epidemiológicos pode ser difícil determinar com certeza a causa da perda de determinado dente. Muitas vezes, o próprio indivíduo desconhece ou não se recorda do motivo da perda. Durante a exploração dos dados (dados não mostrados), foi observado que cerca de 55\% das perdas dentárias por outras razões eram referentes a terceiros molares. Estudo prévio verificou que as perdas relacionadas ao código 5 representaram menos de $10 \%$ do total de perdas e estiveram associadas aos indivíduos de renda mais alta, de cor da pele branca e moradores dos municípios com melhores indicadores de desenvolvimento social e econômico ${ }^{20}$. Por outro lado, prevalência significativamente maior de perda por cárie foi verificada entre adultos com mais baixa escolaridade $^{19}$. Estes dados reforçam que perdas por cárie e por outra razão possuem perfis diferentes de acometimento, sugerindo que a perda por outra razão seja mais elitizada, decorrente predominantemente de problemas funcionais, como indicações ortodônticas, oclusais ou estéticas sobre as quais os hábitos, comportamentos individuais, escolaridade e condições socioeconômicas parecem exercer pouca ou nenhuma 
Tabela 3. Continuação

\begin{tabular}{|c|c|c|c|c|}
\hline & \multicolumn{2}{|c|}{$\begin{array}{c}\text { Modelo } 3 \\
(\text { Blocos } 1,2 \text { e } 3)\end{array}$} & \multicolumn{2}{|c|}{$\begin{array}{c}\text { Modelo } 4 \\
(\text { Blocos } 1,2,3 \text { e } 4)\end{array}$} \\
\hline & $\beta(\mathbf{E P})$ & Valor $\mathbf{p}$ & $\beta(\mathbf{E P})$ & Valor $\mathrm{p}$ \\
\hline \multicolumn{5}{|c|}{ Bloco 1 - Demográficas e socioeconômicas } \\
\hline Idade (em anos) & $0,66(0,08)$ & 0,000 & $0,61(0,08)$ & 0,000 \\
\hline Anos de estudo & $-0,58(0,06)$ & 0,000 & $-0,50(0,06)$ & 0,000 \\
\hline Renda per capita (em reais) & n.s. & n.s. & n.s. & n.s. \\
\hline \multicolumn{5}{|l|}{ Sexo } \\
\hline Masculino & ref. & & ref. & \\
\hline Feminino & $1,42(0,49)$ & 0,004 & $1,87(0,49)$ & 0,000 \\
\hline \multicolumn{5}{|c|}{ Bloco 2 - Utilização dos serviços odontológicos } \\
\hline \multicolumn{5}{|l|}{ Tipo de Serviço } \\
\hline Privado & ref. & & & \\
\hline Público & $1,12(0,53)$ & 0,037 & n.s. & n.s. \\
\hline \multicolumn{5}{|c|}{ Tempo desde a última consulta (anos) } \\
\hline \multicolumn{5}{|l|}{$<1$} \\
\hline $1-2$ & n.s. & n.s. & n.s. & n.s. \\
\hline$>3$ & n.s. & n.s. & n.s. & n.s. \\
\hline \multicolumn{5}{|l|}{ Uso regular/periódico } \\
\hline \multicolumn{5}{|l|}{ Sim } \\
\hline Não & n.s. & n.s. & n.s. & n.s. \\
\hline \multicolumn{5}{|c|}{ Bloco 3 - Acesso a informações em saúde bucal } \\
\hline \multicolumn{5}{|c|}{ Informações sobre como higiene bucal ${ }^{\#}$} \\
\hline Sempre/Frequentemente & ref. & & ref. & \\
\hline Ocasionalmente & $1,80(0,68)$ & 0,008 & $1,41(0,62)$ & 0,056 \\
\hline Raramente/Nunca & $2,91(0,65)$ & 0,000 & $2,15(0,59)$ & 0,000 \\
\hline \multicolumn{5}{|l|}{ Bloco 4 - Comportamentais } \\
\hline \multicolumn{5}{|l|}{$\mathrm{N}^{\circ}$ escovações diárias } \\
\hline$>3$ & & & ref. & \\
\hline 2 & & & $1,63(0,55)$ & 0,050 \\
\hline$<1$ & & & $2,97(1,09)$ & 0,007 \\
\hline \multicolumn{5}{|l|}{ Uso de fio dental } \\
\hline Usa & & & ref. & \\
\hline Não usa & & & $2,39(0,52)$ & 0,000 \\
\hline $\mathrm{R}^{2}$ ajustado & $24,5 \%$ & & 23,6 & \\
\hline
\end{tabular}

ref. : categoria de referência. n.s. : não significativo.

influência. Possivelmente, as perdas dentárias decorrentes da doença periodontal sejam subestimadas no código 5, em função da impossibilidade de identificação do real motivo da perda entre aqueles que perderam muitos dentes. Assim, a perda por cárie parece apresentar, de forma mais prevalente, a perda decorrente de doença, refletindo o contexto social e os comportamentos dos indivíduos. Por isso, optou-se neste estudo por adotar a perda dentária por cárie (código 4) como variável dependente.

Embora a maioria dos indivíduos $(83,2 \%)$ já tenha experimentado pelo menos uma perda por cárie, o número total destas foi heterogêneo (Figura 2), sendo que poucos concentraram muitas perdas e a maior parte tenha perdido poucos dentes, conforme também observado entre indivíduos de 40-49 anos do Rio Grande do Sul, onde se observou média de $10,2(\mathrm{EP}=0,8)$ perdas dentárias entre indivíduos dentados ${ }^{14}$.

A abordagem hierarquizada permitiu avaliar a importância de cada bloco de variáveis sobre a perda dentária por cárie. A ordem de entrada dos blocos, determinada por teorias e modelos prévios $^{12,17,25,26}$, envolveu conhecidos fatores associados à perda dentária, com exceção do bloco 
3, de Acesso a informações em saúde bucal, cuja relação com a perda dentária parece ter sido pouco explorada na literatura. Seu posicionamento no modelo hierarquizado (na terceira posição) foi coerente com a verificação de que o acesso à informação em saúde bucal seja um mediador entre a utilização dos serviços e o total de perdas dentárias, assim como os comportamentos mostraram-se mediadores entre o acesso a informação e as perdas dentárias. O conceito de mediação implica suposição de relacionamentos causais entre as variáveis envolvidas. Uma variável mediadora é aquela que, ao estar presente na equação de regressão, diminui a magnitude do relacionamento entre uma variável antecedente e uma variável dependente ou critério ${ }^{27}$. Vale comentar a opção pelo enquadramento da variável uso regular/periódico dos serviços odontológicos como variável relacionada ao serviço e não como um comportamento ${ }^{12}$. No Brasil a utilização de tais serviços é mais uma questão relacionada ao acesso do que de hábito, preferência ou comportamento ${ }^{33}$.

Maior número de perdas dentárias entre os mais velhos ${ }^{17,26}$, com menos anos de estudo ${ }^{17,21,26}$ e entre mulheres ${ }^{14,17,34}$ já havia sido verificado em estudos prévios. A relação entre higiene bucal e perdas dentárias também já é estabelecida na literatura ${ }^{12,26}$. Diferindo de outros trabalhos, a cor da pele autodeclarada ${ }^{20}$, a renda ${ }^{14,17,19}$, e o hábito tabagista ${ }^{14,26}$ não se mantiveram associada às perdas na presente investigação. Outros estudos nacionais conduzidos entre adultos também não encontraram associação da perda dentária com a cor da pele ${ }^{14,17}$. Já a renda, perdeu sua significância ao ser controlada pelas variáveis de Utilização dos serviços. Possivelmente isso tenha ocorrido em virtude da baixa renda per capita relatada pela grande maioria da população investigada, tornando-a de certa forma homogênea quanto à renda. Quanto ao tabaco, a ausência dessa relação também já foi encontrada previamente ${ }^{35}$. Nenhuma variável do bloco referente à Utilização dos serviços odontológicos manteve-se associada com o total de perdas dentárias após ajuste pelos outros blocos de variáveis. Outros estudos também verificaram associação entre uso dos serviços odontológicos e perdas dentárias somente nas análises univariadas, não permanecendo associadas nos modelos múlti$\operatorname{plos}^{14,20,35}$, sugerindo que sofrem efeito de variáveis de confundimento.

Dentre as variáveis relativas ao Acesso a informações em saúde bucal, acesso a informações sobre como evitar problemas bucais e sobre dieta não permaneceram associadas às perdas dentárias por cárie, ainda quando controladas internamente dentro do bloco 3. A pergunta sobre 0 acesso a informações de como evitar problemas bucais foi realizada de forma abrangente, impossibilitando inferências sobre a procedência de tais informações. A ausência de associação relativa a informações sobre dieta não surpreende, pois pela baixa frequência com que o serviço tem fornecido informações sobre dieta seria difícil esperar qualquer impacto advindo desta prática.

O principal achado desta análise refere-se à relação linear entre a frequência do acesso a informações sobre higiene bucal e o número total de perdas dentárias por cárie entre adultos, mesmo após ajuste pelos demais blocos de variáveis. Adultos que raramente/nunca receberam informações sobre higiene bucal perderam maior número de dentes por cárie do que aqueles que sempre/frequentemente receberam tais informações $(\mathrm{p}=0,000)$. Já entre os que receberam tais informações ocasionalmente, também quando comparados aos que as receberam sempre/frequentemente, a relação mostrou valor de $\mathrm{p}$ limítrofe ( $\mathrm{p}$ $=0,056$ ). Estudo prévio evidenciou efeito temporário de abordagens educativas na redução da placa bacteriana ${ }^{9}$, o que poderia justificar essa relação linear, sugerindo que a frequência de reforço é importante e deve ocorrer ao longo da vida, não somente na idade adulta. A educação em saúde tem sido praticamente inerente à assistência odontológica há quase um século. As intervenções adotadas neste sentido variam consideravelmente, desde a simples disposição de informação até a utilização de complexos programas que envolvam estratégias de mudança de comportamento ${ }^{9}$. Apesar do presente estudo não ter investigado as metodologias adotadas, ainda assim foi possível identificar que o simples acesso a informação sobre higiene bucal, possivelmente contemplando técnicas de escovação e uso de fio dental, tenha impacto sobre o total de perdas dentárias. Este achado enfatiza a importante responsabilidade ética e social da Odontologia em garantir maior repasse de informações adequadas sobre cuidados em saúde bucal à população em geral.

Vale ainda ressaltar que pela forma como a pergunta foi realizada (Recebeu orientações sobre higiene bucal nos serviços odontológicos?) a questão da oferta de informações é trazida para o âmbito dos serviços, indicando a importância do mesmo na orientação de seus usuários ao longo da vida. Sabe-se que disparidades no acesso e uso dos serviços podem contribuir com iniquidades em saúde ${ }^{12}$. Indivíduos pouco informados 
parecem usar os serviços odontológicos de forma predominantemente pontual, frente a uma condição dentária precária ou de urgência onde tratamentos restauradores podem não ser mais viáveis ${ }^{36}$, aumentando a probabilidade de receberem extrações. Estudo longitudinal verificou que a presença alguma doença no elemento dentário foi o principal fator associado à perda de dentes após 24 meses de seguimento, e o afastamento dos serviços odontológicos, com seu uso pontual, esteve associado à maior incidência de perda dentária ${ }^{25}$. Por outro lado, foi observado que mesmo o acesso a tratamento restaurador pode fracassar em evitar a perda dentária se for desprovido de ênfase à prevenção da progressão e surgimento de novas lesões de cárie ${ }^{13}$. Assim, sugere-se que tanto em um contexto de atendimento de urgência, quanto de tratamento sequencial, a oferta de informações sobre higiene bucal proveniente dos serviços seja relevante.

Com a exceção dos raros casos de autoextração dentária, a única maneira de experimentar perdas dentárias é tendo utilizado os serviços odontológicos. Neste sentido o grau em que informações e opções de tratamento são discutidos com pacientes ao utilizarem serviços odontológicos também desempenham um papel em iniquidades sociais na perda do dente. Gilbert et al. ${ }^{12}$ verificaram, em 48 meses de acompanhamento, que indivíduos afro-americanos e com baixo status socioeconômico foram menos propensos a utilizarem serviços odontológicos, mas quando utilizaram tiveram menor possibilidade de receber tratamentos alternativos à extração, receberam menos informações sobre seus tratamentos ou soluções alternativas, relataram menor conhecimento de certos tratamentos que poderiam prevenir a perda de dente, ainda que diante da mesma extensão e severidade da doença em brancos não-hispânicos ${ }^{12}$. Assistência em saúde revestida de discriminação ou preconceito velado, apenas reproduz, dentro do âmbito dos serviços de saúde, a exclusão social. Os serviços devem contribuir com o principio da equidade, e conferir ao ambiente clínico maiores oportunidades de aprendizado, procurando assegurar a todos o acesso aos recursos necessários para que cuidados odontológicos sejam efetivamente, um direito humano ${ }^{37}$.

O ambiente clínico apresenta uma oportunidade de aprendizagem ${ }^{1}$. Neste sentido, chamou atenção e preocupou a alta prevalência $(63,1 \%)$ dos que relataram nunca/raramente ter recebido informações sobre dieta nos serviços odontológicos. O serviço precisa se responsabilizar por isso. É um contra censo dentro do contexto de promoção de saúde ${ }^{38}$, que os serviços odontológicos ainda não estejam assumindo seu papel dentro da abordagem de fatores de risco comum, nem mesmo garantindo simples acesso a informações relativas à dieta.

O fracasso de programas educativos do passado em impactar determinantes sociais e econômicos da saúde pode ter contribuído para a redução da informação no contexto da promoção de saúde contemporânea. Esses fracassos podem ter conduzido a uma subestimação do potencial papel da educação em saúde na abordagem de determinantes sociais. A "alfabetização em saúde" (health literacy) é um conceito relativamente novo em promoção de saúde, usado para descrever os resultados das atividades de educação e comunicação em saúde, enfocando melhor acesso das pessoas às informações em saúde e na capacidade dos indivíduos de usar tais informações eficientemente, sendo base para o empowerment ${ }^{38}$. É neste contexto que a interpretação de nossos achados deve ser encarada. Os serviços precisam, cada vez mais, oferecer informação de qualidade acompanhada de conteúdos políticos da educação em saúde, focados sobre melhorar a bagagem das pessoas em superar barreiras estruturais da saúde. O desenvolvimento de habilidades e atitudes precisa ser conducente à aquisição de poder técnico (saberes) e político para atuar em prol de sua saúde ${ }^{39}$.

O percentual da variância explicado pelo modelo hierarquizado final (modelo 4) foi modesto $\left(\mathrm{R}^{2}\right.$ ajustado $\left.=27,5 \%\right)$, em grande parte porque a perda dentária representa um desfecho complexo e multifatorial, e é provável que importantes variáveis explicativas não tenham sido incluídas nas análises. Estudo com três anos de segmento que considerou variáveis sociais, clínicas e comportamentais na predição da perda dentária, chegou ao modelo final com $29 \%$ de sensibilidade ${ }^{35}$. É importante compreender que os fatores que entram no modelo não são causas diretas da perda dentária, na realidade apenas refletem aspectos de um processo complexo, cujo resultado pode ser a perda de um ou mais dentes. Além disso, esses aspectos retrataram características dos indivíduos que perderam dentes ao invés das características dos dentes que foram perdidos ${ }^{35}$. Tudo isso indica que apenas uma compreensão parcial da perda de dentes pode ser alcançada através de estudos epidemiológicos deste tipo.

O plano amostral, a calibração dos examinadores, a coleta de dados registrada em computador de mão e a condução das análises conside- 
rando a correção pelo efeito de desenho foram estratégias que garantiram validade e confiabilidade aos dados. O número de adultos que compôs a amostra avaliada neste estudo foi maior que o mínimo previsto, mesmo após a exclusão de algumas categorias por sua pequena representatividade. O efeito de desenho (Deff) de 1,38 sofrido pela variável dependente foi inferior aos 2,0 estimados no plano amostral, garantindo poder de inferência para esta variável. Porém, o presente estudo apresenta algumas limitações. Primeiramente por tratar-se de estudo transversal, ou seja, o acesso a informações em saúde bucal e o total de perdas dentárias foram medidos num mesmo momento, não sendo possível determinar a sequência temporal dos eventos. Além disso, conforme já comentado, é difícil na prática de levantamentos epidemiológicos determinar com certeza se o dente foi de fato perdido por cárie, ainda que tenhamos tentado restringir para minimizar vieses. Assim, estudos longitudinais ou ensaios randomizados poderão garantir maiores esclarecimentos sobre a questão.

A violação de dois dos cinco pressupostos preconizados para a realização de regressão linear não parece tão grave, pois já foi sugerido que a regressão múltipla seja eficaz contra a violação de grande parte dos pressupostos ${ }^{27}$. Outros estudos também realizaram regressão linear tendo como variável dependente o número de dentes presentes ou ausentes ${ }^{21,25,26,34}$, embora não tenham apresentado informações sobre os testes de pressupostos. A regressão linear é indicada para variáveis dependentes numéricas e oferece a vantagem de observar a magnitude do impacto na variável dependente com a alteração de uma unidade na variável independente, o que torna a análise mais sensível. A violação do pressuposto da homocedasticidade em termos práticos enfraquece, mas não invalida a análise. Já a violação do pressuposto da normalidade pode ser ate- nuada por meio do aumento do tamanho da amostra da população pesquisada ${ }^{27}$.

\section{Conclusão}

A alta prevalência de perdas dentárias entre adultos aponta para a necessidade de programas direcionados ao atendimento dessa parcela populacional pelos serviços odontológicos, considerando medidas preventivas e educativas em idades mais precoces. As perdas dentárias por cárie, que representaram de forma mais prevalente a perda decorrente de doença, mostraram-se relacionadas a contextos desfavoráveis: pobreza, desinformação, pouco cuidado consigo mesmo, ou mesmo por parte dos serviços. O acesso a informações sobre higiene bucal proveniente dos serviços odontológicos mostrou relação linear com o total de perdas dentárias, mesmo após ajuste por variáveis demográficas e socioeconômicas, de utilização dos serviços odontológicos e comportamentais. A frequência com que informações e opções de tratamento são discutidos com pacientes parecem desempenhar um papel em iniquidades sociais na perda do dente. Além disso, indivíduos pouco informados parecem usar os serviços odontológicos já diante de uma condição dental mais precária, apresentando maior probabilidade de receber uma extração. Chamou atenção a baixa frequência de oferta de informações sobre dieta pelos serviços odontológicos, e dessa forma, tais informações não estiveram associadas ao total de perdas dentárias. Sugere-se maior valorização da interação entre profissionais e usuários, incentivando-os a trocar conhecimentos. Os serviços odontológicos precisam garantir melhor acesso das pessoas às informações em saúde no contexto de promoção de saúde, tendo o conhecimento como pano de fundo para a busca de maior equidade em saúde bucal. 


\section{Colaboradores}

DS Haikal atuou na concepção do estudo, realizou as análises, a interpretação dos dados e a redação do artigo. AMEBL Martins atuou na concepção do estudo, coordenou o trabalho de campo e o processamento dos dados, além de contribuir na análise e revisão crítica do artigo. PHS Aguiar contribuiu na análise, interpretação dos dados e na redação do artigo. MF Silveira e AMB De-Paula contribuíram na análise, interpretação dos dados e revisão crítica do artigo. EF Ferreira contribuiu na concepção, interpretação e na revisão crítica do estudo.

\section{Agradecimentos}

Agradecemos o apoio logístico da Unimontes e da Prefeitura Municipal de Montes Claros, o fomento da FAPEMIG e a colaboração de todos os participantes.

\section{Referências}

1. Green LW, Kreuter MW. Health promotion planning: an educational and environmental approach. Mountain View: Mayfield Publishing Company; 1991.

2. Coulter A, Ellins J. Effectiveness of strategies for informing, educating, and involving patients. $\mathrm{Br} \mathrm{Med}$ J 2007; 335(7609):24-27.

3. Brown T, Summerbell C. Systematic review of schoolbased interventions that focus on changing dietary intake and physical activity levels to prevent childhood obesity: an update to the obesity guidance produced by the National Institute for Health and Clinical Excellence. Obes Rev 2009; 10(1):110-141.

4. McPherson CJ, Higginson IJ, Hearn J. Effective methods of giving information in cancer: a systematic literature review of randomized controlled trials. J Public Health Med 2001; 23(3):227-234.

5. Cleland CL, Tully MA, Kee F, Cupples ME. The effectiveness of physical activity interventions in socio-economically disadvantaged communities: A systematic review. Prev Med 2012; 54(6):371-380.

6. Murray E, Burns J, See TS, Lai R, Nazareth I. Interactive health communication applications for people with chronic disease. Cochrane Database Syst Rev 2005; 19(4):32-34.

7. Wagner EH, Beery WL, Schoenbach VJ, Graham RM. An assessment of health hazard/health risk appraisal. Am J Public Health 1982; 72(4):347-352.

8. Schoenbach VJ, Wagner EH, Beery WL. Health risk appraisal: review of evidence for effectiveness. Health Serv Res 1987; 22(4):553-580.

9. Kay EJ, Locker D. Is dental health education effective? A systematic review of current evidence. Community Dent Oral Epidemiol 1996; 24(4):231-235.

10. Ferrazzano GF, Cantile T, Sangianantoni G, Ingenito A. Effectiveness of a motivation method on the oral hygiene of children. Eur J Paediatr Dent 2008; 9(4):183-187.

11. Macnab A, Kasangaki A. 'Many voices, one song': a model for an oral health programme as a first step in establishing a health promoting school. Health Promot Internation 2012; 27(1):63-73.

12. Gilbert GH, Duncan RP, Shelton BJ. Social Determinants of Tooth Loss. Health Serv Res 2003; 38(6 Pt 2):1843-1862.

13. Caldas Júnior AF, Silveira RCJ, Marcenes W. The impact of restorative treatment on tooth loss prevention. Pesqui Odontol Bras 2003; 17(2):166-170.

14. Susin C, Oppermann RV, Haugejorden O, Albandar JM. Tooth loss and associated risk indicators in an adult urban population from south Brazil. Acta Odontol Scand 2005; 63(2):85-93.

15. Vargas AMD, Paixão HH. Perda dentária e seu significado na qualidade de vida de adultos usuários de serviço público de saúde bucal do Centro de Saúde Boa Vista em Belo Horizonte. Cien Saude Colet 2005; 10(4):1015-1024.

16. Polzer I, Schwahn C, Völzke H, Mundt T, Biffar R. The association of tooth loss with all-cause and circulatory mortality. Is there a benefit of replaced teeth? A systematic review and meta-analysis. Clin Oral Investig 2012; 16(2):333-351. 
17. Barbato PR, Nagano HCM, Zanchet FN, Boing AF, Peres MA. Perdas dentárias e fatores sociais, demográficos e de serviços associados em adultos brasileiros: uma análise dos dados do Estudo Epidemiológico Nacional (Projeto SB Brasil 2002-2003). Cad Saude Publica 2007; 23(8):1803-1814.

18. McCaul LK, Jenkins WMM, Kay EJ. The reasons for extraction of permanent teeth in Scotland: a 15year follow-up study. Br Dent J 2001; 190(12):658662.

19. Jovino-Silveira RC, Caldas Jr AF, Souza EH, Gusmão ES. Primary reason for tooth extraction in a Brazilian adult population. Oral Health Prev Dent 2005; 3(3):151-157.

20. Barbato PR, Peres MA. Perdas dentárias em adolescentes brasileiros e fatores associados: estudo de base populacional. Rev Saude Publica 2009; 43(1):13-25.

21. Starr JM, Pattie A, Whalley LJ, Deary IJ. Predictors of tooth loss in the 1921 Lothian Birth Cohort. Age Ageing 2008; 37(1):111-114.

22. Martins AMEBL, Guimarães ALS, De-Paula AMB, Pires CPAB, Haikal DS, Silva JM, Silveira MF, Caldeira TCR, Eleutério NB, Silveira AM, Almeida BM, Almeida CM, Freitas CV, Botelho DMM, Chaves KTS, Pereira PMB, Lima RS, Pereira SM, Silva TF, Duarte VM, Silva VEP, Pordeus IA. Levantamento epidemiológico das condições de saúde bucal da população de Montes Claros - Projeto SBMOC. Rev Unimontes Cientifica 2012; 14(1):3-14.

23. Organização Mundial de Saúde (OMS). Oral Health surveys: basic methods. $4^{\text {th }}$ Edition. Geneva: ORH, EPID; 1997.

24. Cicchetti DV, Volkmar F, Sparrow SS, Cohen $\mathrm{D}$, Fermanian J, Rourke BP. Assessing the reliability of clinical scales when the data have both nominal and ordinal features: proposed guidelines for neuropsychological assessments. J Clin Exp Neuropsychol 1992; 14(5):673-686.

25. Gilbert GH, Miller MK, Duncan RP, Ringelberg ML, Dolan TA, Foerster U. Tooth-specific and person-level predictors of 24-month tooth loss among older adults. Community Dent Oral Epidemiol 1999; 27(5):372-385.

26. Sun-Young C, Keun-Bae S, Sang GL, Youn-Hee C. The strength of age effect on tooth loss and periodontal condition in Korean Elderly. Arch Gerontol Geriatr 2011; 53(2):243-248.

27. Abbad G, Torres CV. Regressão múltipla stepwise e hierárquica em Psicologia Organizacional: aplicações, problemas e soluções. Estud Psicol 2002; 7(4):19-29.
28. Field A. Descobrindo a Estatística Utilizando o SPSS. Porto Alegre: Artmed; 2009.

29. Brasil. Ministério da Saúde (MS). Projeto SB Brasil 2003: condições de saúde bucal da população brasileira 2002-2003: resultados principais. Brasília: MS; 2004.

30. Brasil. Ministério da Saúde (MS). Projeto SB Brasil 2010: Resultados principais. Brasília: MS; 2011.

31. World Health Organization (WHO). Collaborating Centre for Oral Health Services Research University College Cork. Oral Health of Irish Adults 2000 2002. Cork: WHO; 2007.

32. Cimões R, Caldas Júnior AF, Souza EHA, GusmãoES. Influência da classe social nas razões clínicas das perdas dentárias. Cien Saude Colet 2007; 12(6): 1691-1696.

33. Pinheiro RS, Viacava F, Travassos CB, Santos A. Gênero, morbidade, acesso e utilização de serviços de saúde no Brasil. Cien Saude Colet 2002; 7(4):687-707.

34. Copeland LB, Krall EA, Jackson Brown L, Garcia R, Streckfus CF. Predictors of Tooth Loss in Two US Adult Populations. J Public Health Dent 2004; 64(1):31-37.

35. Locker D, Ford J, Leake JL. Incidence of and risk factors for tooth loss in a population of older Canadians. J Dent Res 1996; 75(2):783-789.

36. Hiramatsu DA, Tomita NE, Franco LJ. Perda dentária e a imagem do cirurgião-dentista entre um grupo de idosos. Cien Saude Colet 2007; 12(4):10511056.

37. Narvai PC. Collective oral health: ways from sanitary dentistry to buccality. Rev Saude Publica 2006; 40(no Esp.):141-147.

38. Nutbeam D. Health literacy as a public health goal: A challenge for contemporary health education and communication strategies into the $21^{\text {st }}$ century. Health Promot Internation 2000; 15(3):259-267.

39. Pereira ALF. Pedagogical approaches and educational practices in health sciences. Cad Saude Publica 2003; 19(5):1527-1534.

Artigo apresentado em 03/09/2012

Aprovado em 24/10/2012

Versão final apresentada em 09/11/2012 